\title{
A graded Bayesian coherence notion
}

\author{
Frederik Herzberg \\ Copyright notice: The final publication, in ERKenntnis, 79 (2014), no. 4, \\ pp. 843-869, is available at link. springer.com, \\ doi:10.1007/s10670-013-9569-6
}

\begin{abstract}
Coherence is a key concept in many accounts of epistemic justification within 'traditional' analytic epistemology. Within formal epistemology, too, there is a substantial body of research on coherence measures. However, there has been surprisingly little interaction between the two bodies of literature.

The reason is that the existing formal literature on coherence measure operates with a notion of belief system that is very different from - what we argue is - a natural Bayesian formalisation of the concept of belief system from traditional epistemology. Therefore, formal epistemology has so far only been concerned with one particular - arguably not even very natural way of formalising coherence of belief systems; it has by no means refuted the viability of coherentism. In contrast to the existing literature, we formalise belief systems as families of assignments of (conditional) degrees of belief (which may be compatible with several subjective probability measures).

Within this framework, we propose a Bayesian formalisation of the thrust of BonJour's coherence concept in The structure of empirical knowledge (1985), using a combination of Bayesian confirmation theory and basic graph theory. In excursions, we introduce graded notions for both logical and probabilistic consistency of belief systems - the latter being based on certain geometrical structures induced by probabilistic belief systems.

For illustration, we reconsider BonJour's "ravens" challenge (op. cit., p. 95f.). Finally, potential objections to our proposed formal coherence notion are explored.

Key words: epistemic justification; coherentism (epistemology); coherence measure; Laurence BonJour; Bayesianism; Bayesian confirmation theory; connectivity (graph theory); Hausdorff measure
\end{abstract}

Institut für Mathematische Wirtschaftsforschung, Universität Bielefeld, Universitätsstraße 25, D-33615 Bielefeld, Germany. fherzberg@uni-bielefeld.de. 
Contents

\begin{tabular}{|c|c|c|}
\hline \multicolumn{3}{|c|}{ 1. Introduction and motivation } \\
\hline & Coherence measures vs. Bayesian formalisations & \\
\hline & traditional coherence concepts & \\
\hline 3. & A formal Bayesian framework for belief systems & 6 \\
\hline 4. & BonJour's coherence notion & 7 \\
\hline 5. & A formalisation of BonJour's coherence concept & 8 \\
\hline 6. & Examples. BonJour's challenge & 17 \\
\hline 7. & Discussion & 24 \\
\hline & rences & 27 \\
\hline
\end{tabular}

\section{Introduction and motivation}

Coherence is a key concept in 'traditional' (as opposed to formal) analytic epistemology. There is a whole family of epistemological positions (usually subsumed under the heading of 'coherentism') according to which the coherence of a belief system plays a pivotal rôle — sometimes even as a necessary and sufficient condition in determining whether an agent is epistemically justified in having the belief system in question. In addition, even some moderate foundationalists (Audi [5], for instance) would concede that coherence increases - or constitutes even a necessary condition for — the epistemic justifiability of a belief system.

Moreover, belief systems may be more or less coherent; that coherence is a matter of degree has long been advocated, especially by coherentists (cf. e.g. Brendel [12]). To be sure, formal epistemologists have invested considerable energy into finding suitable measures of coherence (cf. e.g. Shogenji [66 for a relatively early and well-known proposal). Various authors have also shown that no coherence measure can satisfy certain sets of desiderata (e.g. Klein and Warfield [43, 44]). While there are arguably reasons to view some of those "impossibility results" rather critically (cf. e.g. Meijs and Douven [52] and Schupbach 65), a large set of proposed coherence measures has just recently been re-examined with respect to a new, arguably intuitive criterion - and been found wanting (cf. Siebel and Wolff [67]).

It would therefore probably be fair to say that while the literature on coherence measures is not unanimous in its disillusionment with formal coherence notions, it does prima facie cast a shadow of doubt on any epistemological theory that heavily depends on the notion of coherence. In particular, this applies to coherentist theories of doxastic or more generally epistemic justification.

The purpose of the present paper is to demonstrate that coherentist theories of doxastic justification have not much to fear from the literature on coherence measures because (1) coherence measures 
merely formalise a rather special notion of coherence of belief systems to which coherentists are by no means committed and (2) a formal definition of a Bayesian coherentist concept of coherence is feasible.

In order to show this, we will first (in Section 2p argue that (1) the formal literature on coherence measures in general operates with a concept of belief systems that is quite foreign to a straightforward Bayesian formalisation of belief systems and is therefore concerned with a rather peculiar coherence notion. In particular, the concept of coherence investigated by the formal literature on coherence measures cannot cover natural Bayesian formalisations of coherence concepts from traditional epistemology.

On a more constructive note, we shall thereafter (2) propose a natural formal (Bayesian) framework for the analysis of belief systems (Section 3). In contrast to the existing literature on coherence measures, we view belief systems not as sets of propositions, but as families of assignments of (conditional) degrees of belief which may be compatible with several subjective probability measures.

Within this framework, then, we shall outline a formalisation of Laurence BonJour's [6] coherence concept (Sections 4 and 5). At the heart of this formalisation is a vector-valued (multi-dimensional) coherence notion whose components are formalisations of each of the first four of BonJour's desiderata for a coherence notion. It is plausible that the weight given to each of BonJour's desiderata depends on the epistemic context. Weighted averaging (perhaps corrected by monotone transformations) in accordance with the respective epistemic context yields a single-valued coherence measure. The multidimensional coherence concept at the centre of this construction will involve a notion of degree of confirmation from Bayesian confirmation theory as well as a graph-theoretic notion of connectivity. In two excursions, we introduce a graded notion of probabilistic consistency of belief systems based on the geometrical structure of certain sets of probability measures, as well as a graded notion of logical consistency based on the parametric structure of the Lockean thesis (according to which an individual has full belief in a proposition $S$ if and only if he holds partial belief in $S$ with sufficiently high degree, say $c \gg 1 / 2$ ).

We shall illustrate the formal machinery by means of two benchmark examples of belief systems from the literature (Section 6): Just as Bovens and Hartmann [9] did, we submit our proposed coherence measure to the test of "BonJour's challenge". Several potential objections to our proposal for a new graded coherence notion are — along with possible refutations — explored in Section 7. 


\section{Coherence measures vs. Bayesian formalisations of traditional coherence concepts}

For much more than a decade, formal epistemologists have directed considerable efforts to finding formal, mathematical measures of what they viewed as - coherence 1 Several authors have also shown that no coherence measure can satisfy certain sets of desiderata..$^{2}$

Now, an (almost) ubiquitous feature of the formal literature on coherence measures is that belief systems (information sets) are conceived as collections of (binary assignments of truth values to) propositions, rather than as assignments of degrees of belief to (conditional) events. The literature on coherence measures accommodates uncertainty only by allowing for varying degrees of reliability in the sources of the propositions in the information set (while assuming the information sources to be probabilistically independent), but it does not allow for uncertainty at a more fundamental level..$^{3}$ Frequently, the information sets (sets of belief) under consideration in the literature on coherence measures are presented as sets of "witness reports", which makes this kind of setup defensible for certain purposes.

As a representative example, consider the framework in the paper by Moretti and Akiba [55], which is not only of substantial historical interest, as it examines C.I. Lewis' 49] view of coherence among others, but also reviews several of the better-known coherence measures. The framework of that paper presupposes an objectively given probability measure $\operatorname{Pr}$ on some algebra $\mathcal{A}$ of propositions; a "set of beliefs" is a subset of $\mathcal{A}$, and a coherence measure is a function from a (subset of the) power-set of $\mathcal{A}$ to the reals. For example, Shogenji's [66] coherence measure is defined (using the set-theoretic symbols for the Boolean operations in $\mathcal{A}$ ) as

$$
C_{S}\left(\left\{B_{1}, \ldots, B_{n}\right\}\right)=\frac{\operatorname{Pr}\left(B_{1} \cap \cdots \cap B_{n}\right)}{\operatorname{Pr}\left(B_{1}\right) \times \cdots \times \operatorname{Pr}\left(B_{n}\right)}
$$

\footnotetext{
${ }^{1}$ For some well-known papers, cf. e.g. Shogenji [66], Akiba [1] and Fitelson [25 - as well as Dietrich and Moretti [19] for a systematic comparison.

${ }^{2}$ Cf. e.g. Klein and Warfield [43, 44, Bovens and Hartmann [8, 10, 11] and Olsson [56, [57, 58]; for a critical assessment of those "impossibility results", cf. Meijs and Douven [52] and Schupbach 65. That coherence measures generally fail to satisfy a rather intuitive criterion has also been demonstrated by Siebel and Wolff 67.

${ }^{3}$ Douven and Meijs 22 represent an exception to this rule. They propose a quantitative theory of coherence in the framework of a quantitative bootstrap confirmation theory à la Glymour [29]. But in this setting, the theories to be confirmed consist of propositions given some prior. Therefore, even the theories in Douven and Meijs' 22 paper are not belief systems as Bayesians would normally understand them.
} 
for all $B_{1}, \ldots, B_{n} \in \mathcal{A}$ (or, in more economic notation, $C_{S}(\mathbf{E})=$ $\operatorname{Pr}(\bigcap \mathbf{E}) / \prod_{B \in \mathbf{E}} \operatorname{Pr}(B)$ for all $\mathbf{E} \subseteq \mathcal{A}$; this definition allows for coherence to be defined for infinite "sets of beliefs", too).

In contrast to such a view of "sets of beliefs", belief systems as Bayesians understand them in other contexts are systems of conditional probability assignments - corresponding to conditional degrees of belief - , not propositions or binary assignments of truth values to propositions. For a Bayesian, a belief system is generally encoded by a probability measure - or, more generally, by a (possibly singleton) set of probability measures.

We will present a formal framework for an analysis of such belief systems from a Bayesian perspective shortly. At this point, we merely conclude that the existing literature on coherence measures has not addressed the problem of measuring the coherence of belief systems when understood in Bayesian terms ${ }^{4}$ Even though one of the earliest papers on coherence measures (Klein and Warfield [43]) explicitly refers to BonJour's coherentist treatise [6] (as do Bovens and Hartmann [9]), the literature on coherence measures provides by no means a definitive answer about the prospects of coherentism, because it operates within a framework that is arguably not the most natural one.

The aim of the remainder of the paper is now to develop a graded coherence notion for belief systems conceived as sets of conditional probability assignments - in a way that is both mathematically rigorous and takes into account the insights of traditional, nonformal epistemology. More technically speaking, we shall formalise BonJour's [6] coherence concept by showing how to view a belief system (thus understood) as a Bayesian network which induces a geometrical structure on the set of probability measures compatible with the belief system in question. Depending on the epistemic context, some of the desiderata may be more important than others (perhaps even in a non-linear manner), for constituting doxastic justification. In order to arrive at a single-valued coherence measure that respects the respective epistemic context, one can form the weighted average of the components of this vector-valued coherence measure (perhaps after applying monotone transformations that remove non-linearities). The result will satisfy all of BonJour's desiderata.5

\footnotetext{
${ }^{4}$ One may wonder where such an understanding of sets of beliefs is coming from. However, unless one is a Bayesian, it is very natural to identify beliefs with propositions and thus belief sets with sets of propositions. Moreover, as Hansson and Olsson 32 have pointed out, there is a long-standing association of the AGM theory of belief revision due to Alchourrón, Gärdenfors and Makinson [2] with coherentism.

${ }^{5}$ Note that the expression "in proportion" in BonJour's desiderata (II) and (V) need not be understood in a literal manner (as if it required linear dependence). Otherwise, BonJour would have presupposed that coherence-salient qualities of a doxastic system such as the "degree of probabilistic consistency" or the "presence
} 


\section{A formal Bayesian framework for belief systems}

Our formal framework is motivated as follows. We consider an epistemic agent who has to choose one among several competing, in general rather large, belief systems (for instance, entire worldviews). One of the criteria she employs in the choice of a belief system will be relative coherence (ultimately to be defined in Section 5).

We make the 'structural' assumption that all of her candidate belief systems are sets of conditional degrees of belief, and that the pairs of propositions to which the belief systems assign conditional degrees of belief are pairs of propositions from the same algebra. Furthermore, we adopt the thesis of probabilism by assuming that these conditional degrees of belief correspond to conditional probability assignments. ${ }^{6}$ Thus, we essentially subscribe to the assumption that the epistemic agent assigns to any conditional event either a precise degree of belief or none at all..$^{7}$ (A similar construal of belief systems can already be found in Carnap [13].)

Of course, this is a somewhat stylised formal framework. Nevertheless, we have chosen it in order to convey the main idea of our proposed graded coherence notion. In principle, it could be replaced by a more comprehensive set-up - e.g. one that allows for imprecise or interval probabilities ${ }^{8}$ at a fuller scale.

The natural formal description of our stylised framework is of a simple Bayesian kind. While our interpretation of the framework is broadly subjective internalist, the framework itself and the development of the formal coherence notion are independent of that interpretation. (Our subjective internalist interpretation may be read as a mere manner of speaking rather than as the expression of an actual philosophical claim.)

In order to introduce the details of the formal framework, we fix some algebra $\mathcal{A}$ of propositions. (In the realm of interpretation, this should be chosen in such a way as to consist of all propositions about

of unexplained anomalies in the believed content of the system" admit a canonical quantification with values in a vector space.

${ }^{6}$ There is, of course, an ongoing discussion in formal epistemology about how to optimally defend probabilism, cf. e.g. Joyce 39, Easwaran and Fitelson 23, Fitelson and McCarthy [26, Leitgeb and Pettigrew [47, 48, Wedgwood [71; reviewing this discussion is, however, not within the scope of this paper.

${ }^{7}$ We write "essentially" because even this framework will under some conditions entail the assignment of interval probabilities to certain propositions/events: The monotonicity of probability measures always implies $P(B \mid C) \geq \alpha$ for all $\alpha, A, B, C$ with $P(A \mid C)=\alpha$ and $A \subseteq B$. Therefore, whenever $B$ belongs to the algebra generated by the propositions featuring in $\mathcal{S}$ and both $\langle A \mid C \| \alpha\rangle \in \mathcal{S}$ and $A \subseteq B$ holds, then one will have $P(B \mid C) \geq \alpha$ for all $P$ compatible with the belief system $\mathcal{S}$. In other words, any probability measure $P$ compatible with $\mathcal{S}$ will assign a probability $\geq \alpha$ to the conditional event $B$-given- $C$.

${ }^{8}$ In the sense of Walley [69, 70] or Weichselberger [72]. 
which our epistemic agent might possibly form conditional beliefs.) A belief system $\mathcal{S}$ will now be a set of conditional probability assignments, interpreted as conditional degrees of belief, to pairs of propositions (i.e., pairs of elements of $\mathcal{A}$ ): We define a belief system to be a set of triples, by convention denoted $\langle A \mid B \| \alpha\rangle$, where $A, B \in \mathcal{A}$ and $\alpha$ is a real number between 0 and 1 . The expression $\langle A \mid B \| \alpha\rangle$ is to be read as: "Given $B$, the belief system assigns to $A$ a conditional degree of belief of $\alpha . "$ While our epistemological interpretation (via conditional degrees of belief) provides the main reason for taking conditional probability assignments as primitive, it is worthwhile to note that Rényi [62 (and to some extent also de Finetti [16]) actually developed foundations for probability theory with conditional probability functions as the fundamental objects.

Note once again that belief systems, in our terminology, are not just sets of propositions - unlike, for example, in the AGM belief-revision literature [2]. Rather, they are systems of conditional degree-of-belief assignments to propositions from a given algebra. In particular, belief systems are not assumed to satisfy logical completeness of any kind.

A probability measure $P: \mathcal{A} \rightarrow[0,1]$ is said to be compatible with a belief system ${ }^{9} \mathcal{S}$ if and only if $P(A \mid B)=\alpha$ holds for all triples $\langle A \mid B \| \alpha\rangle \in \mathcal{S}$. A belief system $\mathcal{S}$ is said to be probabilistically consisten 10 if and only if there exists a probability measure compatible with $\mathcal{S}$.

The set of all probability measures on $\mathcal{A}$ that are compatible with a given belief system $\mathcal{S}$ will be denoted $\mathcal{P}_{\mathcal{S}}$. Thus, a belief system is probabilistically consistent if and only if $\mathcal{P}_{\mathcal{S}}$ is non-empty. In addition to this notion of probabilistic consistency simpliciter, we will later on introduce a graded notion of probabilistic consistency. This notion will be conceived 'semantically' - in the sense that the degree of probabilistic consistency of a belief system $\mathcal{S}$ will be measured in terms of the size of the 'extension' $\mathcal{P}_{\mathcal{S}}$ of the belief system.

\section{BonJour's coherence notion}

Having formalised the notions of belief sytems and probabilistic consistency simpliciter (of belief systems), let us now consider an agent who has to choose between two belief systems $\mathcal{S}_{1}, \mathcal{S}_{2}$. According to coherentists and moderate foundationalists (e.g., Audi [5]), the coherence of the belief systems should be a - for some, even the decisive factor in determining which belief system to adopt. (Holistic coherentists would demand that only 'comprehensive' belief systems be taken under consideration - belief systems which, in particular,

\footnotetext{
${ }^{9}$ Another possible name for the compatible-with relation would be "support", cf. Herzberg [38. This term, however, is already defined in measure theory, whence we shall not use it in the sense of the compatible-with relation lest we create confusion.

${ }^{10}$ This is what de Finetti [15, 16, 17] already calls coherence.
} 
encode all information that is available to the agent and, moreover, assign degrees of belief to all conditional events whose probability will be involved in the agent's decisions. By this means, standard objections such as the isolation objection lose their force and coherence arguably does becomes the single decisive factor.) This calls for the definition of a graded notion of coherence.

We aim at a definition or explication that (1) is in formal, mathematical terms, and (2) at the same takes into account insights from traditional, i.e. as opposed to formal, epistemology. Among contemporary philosophers, BonJour [6] and Lehrer [45] have been perhaps the two foremost defenders of coherentism in traditional epistemology. BonJour also offers a sketch of a graded coherence notion, which is sufficiently detailed to become the point of departure for a formal reconstruction. He suggests the following "reasonable outline of the concept of coherence" [6, p. 94]:

[(I)] A system of beliefs is coherent only if it is logically consistent.

[(II)] A system of beliefs is coherent in proportion to its degree of probabilistic consistency. $[\ldots]$

[(III)] The coherence of a system of beliefs is increased by the presence of inferential connections between its component beliefs and increased in proportion to the number and strength of such connections.

[(IV)] The coherence of a system of beliefs is diminished to the extent to which it is divided into subsystems of beliefs which are relatively unconnected to each other by inferential connections.

$[\ldots]$

$[(\mathrm{V})]$ The coherence of a system of beliefs is decreased in proportion to the presence of unexplained anomalies in the believed content of the system. [6. Section 5.3, pp. 95, 98, 99]

Our task will now be first of all to find explications, in our formal framework, of these five requirements. Depending on the epistemic context, one can then monotonically transform them and form their weighted average, in order to arrive at a context-specific single-valued coherence measure.

\section{A formalisation of BonJour's coherence concept}

For the subsequent formalisation of BonJour's graded notion of coherence, we shall first assume, for simplicity, that $\mathcal{A}$ is actually a 
finite algebra. Moreover, since any such algebra is isomorphic to a (finite) power-set algebra ${ }^{11}$, we may assume that $\mathcal{A}=2^{\Omega}$. Following common practise in probability theory, one may view $\Omega$ as a set of possible worlds, so that any algebra element $A \in \mathcal{A}$ can be understood at the same time as a proposition and as an event, i.e. the extension of a proposition (viz. the set of worlds in which the proposition holds true). The propositional constants $T, \perp$ of truth and falsity correspond to the maximal and minimal elements of the power-set algebra, viz. $\Omega$ and $\varnothing$, respectively - and the Boolean operations of $\wedge, \vee, \neg$ correspond to the set-theoretic operations $\cap, \cup, \complement$.

In the first and most difficult step, we shall define functions that attempt to quantify each of BonJour's desiderata. The result will be a vector-valued coherence measure with components $\beta_{1}, \ldots, \beta_{4}$. Later on, we will briefly address the question of turning this vector-valued coherence measure into a single-valued one.

5.1. Formalising BonJour's first desideratum. The first requirement (I) presupposes an underlying theory of the relation of (full) beliefs and degrees of belief, which is still the subject of an ongoing intense debate in formal epistemology - to which, among others, Arló-Costa and Parikh [3, Foley [27, Leitgeb [46], Arló-Costa and Pedersen [4 have contributed. The most well-known bridge principle is the Lockean thesis, which says that full belief is (partial) belief to a sufficiently high degree $(c$, say).12

A straightforward interpretation of BonJour's requirement (I) would be to say that a belief system is logically consistent if the set of those elements of $\mathcal{A}$ (propositions/events) to which an unconditional degree of belief $>c$ is assigned by all probability measures compatible with the belief system in question, should not be a logical absurdity i.e., such a system should have a non-empty intersection. This notion is problematic because it hinges on the somewhat arbitrary choice of the parameter $c$.

A very strong version of requirement (I) which circumvents this problem of parameter dependence would be to demand for logical consistency that the intersection of all those propositions/events to which an unconditional degree of belief $>\frac{1}{2}$ is assigned by all probability measures which are compatible with the belief system should be non-empty. In other words, a very strong parameterindependent version of (I) requires:

$$
\eta(\mathcal{S},(1 / 2,1]) \neq \varnothing,
$$

wherein $\eta(\mathcal{S}, I)$, for all $I \subseteq[0,1]$, denotes the intersection of all propositions/events to which a probability within $I$ is assigned by all

\footnotetext{
${ }^{11}$ Cf. e.g. the classical paper by Stone [68, Theorem 12].

${ }^{12}$ Statements that can be interpreted as expressing or at least supporting this thesis occur repeatedly in Locke's Essay [50, Book IV, Chapters XIV-XVII].
} 
probability measures compatible with $\mathcal{S}$ :

$$
\begin{aligned}
\eta(\mathcal{S}, I) & :=\bigcap \bigcap\left\{P^{-1}(I): P \in \mathcal{P}_{\mathcal{S}}\right\} \\
& =\bigcap_{\substack{A \in \mathcal{A} \\
\forall P \in \mathcal{P}_{\mathcal{S}} P(A) \in I}} A .
\end{aligned}
$$

An extremely weak, yet also parameter-independent reading of requirement (I) would only demand for logical consistency that the intersection of all those propositions/events to which an (unconditional) degree of belief $=1$ is assigned by all probability measures which are compatible with the belief system should be nonempty, in symbols:

$$
\eta(\mathcal{S},\{1\}) \neq \varnothing
$$

Both requirements expressed in formulae (1) and (2) take an allor-nothing approach to logical consistency. This is perfectly in line with BonJour's [6] position who viewed logical consistency as a binary concept (see, for example, the comparison of logical and probabilistic consistency quoted below [6, p. 95]). Logical consistency is a binary component of the multi-faceted non-binary, graded concept of coherence.

Now, the strong requirement in formulae (1) seems problematic because on such an interpretation of logical consistency and coherence, the paradoxes of the preface (cf. Makinson [51] and Pollock [59]) or the lottery (cf. e.g. Christensen [14]) would show that rational human beings rather frequently hold utterly incoherent belief systems. Therefore, we propose to formalise logical consistency through the weak requirement in formula (2):

$$
\beta_{1}(\mathcal{S})= \begin{cases}1, & \eta(\mathcal{S},\{1\}) \neq \varnothing \\ 0, & \eta(\mathcal{S},\{1\})=\varnothing .\end{cases}
$$

5.2. Excursion: A graded notion of logical consistency for probabilistic belief systems. Even though this will not enter our formal coherence measure, it is certainly worthwhile to ask if there is a natural graded notion of logical consistency for probabilistic belief systems. And indeed, in view of the parametric nature of the Lockean thesis, there is a candidate for such a concept: One could measure logical consistency through the reciprocal value of the infimal (roughly: minimal) $c$ such that the intersection of all those propositions/events to which an (unconditional) degree of belief $>c$ is assigned by all probability measures which are compatible with the belief system 
becomes non-empty: 13

$$
\tilde{\beta}_{1}(\mathcal{S}):=1 / \inf \{c \in[0,1]: \eta(\mathcal{S},(c, 1]) \neq \varnothing\}
$$

(with the usual conventions: ${ }^{14}$ inf $\varnothing=\infty, 1 / \infty=0,1 / 0=\infty$ ). We have mentioned this possibility of defining a graded notion of logical consistency only for the sake of completeness. However, it is no longer a formalisation of BonJour's first desideratum and we do not suggest that it should be part of a formal coherence concept. The first component of our proposed coherence measure is binary, as will be the second.

5.3. Formalising BonJour's second desideratum. A naïve reading of BonJour's requirement (II) would look for a measure for the degree of probabilistic consistency of a belief system in our above formal framework. However, if one reads this requirement in context, one finds the following paragraph:

Probabilistic consistency differs from straightforward logical consistency in two important respects. First, it is extremely doubtful that probabilistic inconsistency can be entirely avoided. Improbable things do, after all, sometimes happen, and sometimes one can avoid admitting them only by creating an even greater probabilistic inconsistency at another point. Second, probabilistic consistency, unlike logical consistency, is

\footnotetext{
${ }^{13}$ One might perhaps wonder whether $\tilde{\beta}_{1}(\mathcal{S})$ equals the somewhat simpler expression

$$
\bar{\beta}_{1}(\mathcal{S}):=1 / \inf \left\{c \in[0,1]: \exists \omega \in \Omega \bigcap_{\substack{A \in \mathcal{A} \\ \forall P \in \mathcal{P}_{\mathcal{S}} P(A)>c}} A=\{\omega\}\right\} .
$$

To be sure,

$$
\left\{c \in[0,1]: \exists \omega \in \Omega \bigcap_{\substack{A \in \mathcal{A} \\ \forall P \in \mathcal{P}_{\mathcal{S}} P(A)>c}} A=\{\omega\}\right\} \subseteq\left\{c \in[0,1]: \bigcap_{\substack{A \in \mathcal{A} \\ \forall P \in \mathcal{P}_{\mathcal{S}} P(A)>c}} A \neq \varnothing\right\},
$$

so the infimum of the former set is greater or equal than that of the latter. The converse estimate, however, need not hold: For, it may happen that $\bigcap_{\substack{A \in \mathcal{A} \\ \forall \in \mathcal{P}_{S} P(A)>c}} A=\left\{\omega_{1}, \ldots, \omega_{m}\right\}$ with pairwise distinct $\omega_{1}, \ldots, \omega_{m}$ while $P\left\{\omega_{1}\right\}=P\left\{\omega_{i}\right\}$ for all $P \in \mathcal{P}_{\mathcal{S}}$ and $i \leq m$. Then, for any $c^{\prime}<c$ for which $\omega_{1} \notin \bigcap\left\{A \in \mathcal{A}: \forall P \in \mathcal{P}_{\mathcal{S}} P(A)>c^{\prime}\right\}$, one also has $\omega_{i} \notin \bigcap\left\{A \in \mathcal{A}: \forall P \in \mathcal{P}_{\mathcal{S}} P(A)>c^{\prime}\right\}$ for all other $i \leq m$, all the while $\bigcap\left\{A \in \mathcal{A}: \forall P \in \mathcal{P}_{\mathcal{S}} \quad P(A)>c^{\prime}\right\} \subseteq\left\{\omega_{1}, \ldots, \omega_{m}\right\}$ and thus $\bigcap\left\{A \in \mathcal{A}: \forall P \in \mathcal{P}_{\mathcal{S}} \quad P(A)>c^{\prime}\right\}=\varnothing$. For example, if $\mathcal{S}$ is the belief system of ignorance, i.e. $\mathcal{P}_{\mathcal{S}}$ is the singleton consisting of the uniform distribution on a finite set $\Omega$ of states of the world, then $0=\bar{\beta}_{1}(\mathcal{S})<\tilde{\beta}_{1}(\mathcal{S})=1$.

${ }^{14}$ In very liberal belief systems - viz. those that are compatible with several extreme probability measures that assign probability zero to different states of the world - it may happen that $\eta(\mathcal{S},\{1\})=\varnothing$ and therefore a fortiori $\eta(\mathcal{S},(c, 1])=\varnothing$ for all $c$, which entails $\beta_{1}(\mathcal{S})=0$.
} 
plainly a matter of degree, depending on (a) just how many conflicts the system contains and (b) the degree of improbability involved in each case. [6. p. 95]

From a Bayesian perspective, this reasoning seems surprising, to say the least. That events which were a priori (before carrying out) unlikely do sometimes happen and therefore can enter a belief system a posteriori does not at all constitute probabilistic inconsistency, because the small probability was assigned by the prior probability measure, and the high probability was assigned by the posterior probability. For BonJour, this solution is not available because he identifies belief with full belief.

For us, however, there is no reason to copy this view of probabilistic (in)consistency, because in our Bayesian framework, probabilistic consistency is already defined in a very natural way - even though as a binary concept:

$$
\beta_{2}(\mathcal{S})= \begin{cases}1, & P_{\mathcal{S}} \neq \varnothing \\ 0, & P_{\mathcal{S}}=\varnothing\end{cases}
$$

That said, it is still interesting to ask if there exists a sense in which probabilistic consistency could be a matter of degree.

\subsection{Excursion: A graded notion of probabilistic} consistency for probabilistic belief systems. For the construal of a graded notion of probabilistic consistency, we adopt a semantic understanding of 'consistency'. Then, it is most natural to measure the degree of probabilistic consistency of a belief system $\mathcal{S}$ in terms of the "size" of the set $\mathcal{P}_{\mathcal{S}}$ of probability measures compatible with $\mathcal{S}$. While this set will not be finite in general, it is not difficult to realise that the set $\mathcal{P}_{\mathcal{S}}$ possesses a distinctive geometrical structure - at least if the algebra $\mathcal{A}$ is finite.

In order to see this, suppose that $\mathcal{A}$ is finite, so that it can be identified with the power-set algebra of a finite set, $\Omega$, say. Then, on the one hand, the set of probability measures with domain $2^{\Omega}$ can be geometrically represented by the intersection of a hyperplane (consisting of all vectors whose components add up to one) and the unit cube of dimension card $(\Omega)$ : One can canonically — up to permutations of the coordinates - embed the set $\Delta$ of all probability measures defined on $\mathcal{A}$ into $\mathbf{R}^{\text {card }(\Omega)}$ by some map $\iota ; \iota[\Delta]$ will then be the subset of those $\operatorname{card}(\Omega)$-tuples of real numbers whose entries are all between 0 and 1 and sum up to one.

Furthermore, under such a representation, the set $\mathcal{P}_{\{\langle A \mid B \| \alpha\rangle\}}$ is just the intersection of two hyperplanes with the unit cube: If, say, $\Omega=\left\{\omega^{(1)}, \ldots, \omega^{(N)}\right\}, A=\left\{\omega^{(k(1))}, \ldots, \omega^{(k(m))}\right\}$ and $B=$ $\left\{\omega^{(\ell(1))}, \ldots, \omega^{(\ell(n))}\right\}$ for $k:\{1, \ldots, m\} \rightarrow\{1, \ldots, N\}$ and $\ell:$ $\{1, \ldots, n\} \rightarrow\{1, \ldots, N\}$, then $\mathcal{P}_{\{\langle A \mid B \| \alpha\rangle\}}$, the set of probability measures satisfying $P(A \cap B)=\alpha P(B)$, can be represented by the 
set

$$
\iota\left[\mathcal{P}_{\{\langle A \mid B \| \alpha\rangle\}]}=\left\{\begin{array}{c}
x^{(1)}+\cdots+x^{(N)}=1, \\
x \in[0,1]^{N}: \quad \sum_{i \in k[\{1, \ldots, m\}] \cap \ell[\{1, \ldots, n\}]} x^{(i)} \\
=\alpha \sum_{j=1}^{n} x^{(\ell(j))}
\end{array}\right\} .\right.
$$

On the other hand, by definition,

$$
\mathcal{P}_{\mathcal{S}}=\bigcap_{\substack{A, B \in \mathcal{A}, \alpha \in[0,1] \\\langle A \mid B \| \alpha\rangle \mathcal{S}}} \mathcal{P}_{\{\langle A \mid B \| \alpha\rangle\}}
$$

It follows that $\iota\left[\mathcal{P}_{\mathcal{S}}\right]$ itself is just the intersection of the unit cube (of dimension $\log _{2} \operatorname{card}(\mathcal{A})$ ) with several hyperplanes. In other words, for finite algebras $\mathcal{A}$ one can always represent the set of all probability measures that are compatible with a given belief system by a geometrically very well-behaved set, viz. the intersection of a unit cube with finitely many hyperplanes.

This is significant because there is a canonical way of measuring the "size" of such a set, viz. the pair consisting of the Hausdorff dimension and the Hausdorff measure [33]. Since $\iota\left[\mathcal{P}_{\mathcal{S}}\right]$ is a very natural parametric representation of $\mathcal{P}_{\mathcal{S}}$ (in a sense canonical, up to permutation of coordinates — which leaves the Hausdorff measure and Hausdorff dimension unchanged), we have ultimately even found a canonical way of measuring the "size" of $\mathcal{P}_{\mathcal{S}}$ itself.

Thus, one can measure the size of $\mathcal{P}_{\mathcal{S}}$ - and hence the probabilistic consistency of the belief system $\mathcal{S}$ - by the pair consisting of the Hausdorff dimension of the canonical image of $\mathcal{P}_{\mathcal{S}}$ under $\iota$ and its Hausdorff measure:

$$
\tilde{\beta}_{2}(\mathcal{S}):=\left\langle D\left(\iota\left[\mathcal{P}_{\mathcal{S}}\right]\right), \mathcal{H}^{D\left(\iota\left[\mathcal{P}_{\mathcal{S}}\right]\right)}\left(\iota\left[\mathcal{P}_{\mathcal{S}}\right]\right)\right\rangle,
$$

where $\mathcal{P}_{\mathcal{S}}$ is greater than $\mathcal{P}_{\mathcal{S}^{\prime}}$ if and only if either (i) $D\left(\iota\left[\mathcal{P}_{\mathcal{S}}\right]\right)>$ $D\left(\iota\left[\mathcal{P}_{\mathcal{S}^{\prime}}\right]\right)$ or $(\mathrm{ii}) D\left(\iota\left[\mathcal{P}_{\mathcal{S}}\right]\right)=D\left(\iota\left[\mathcal{P}_{\mathcal{S}^{\prime}}\right]\right)$, but $\mathcal{H}^{D\left(\iota\left[\mathcal{P}_{\mathcal{S}}\right]\right)}\left(\iota\left[\mathcal{P}_{\mathcal{S}}\right]\right)>$ $\mathcal{H}^{D\left(\iota\left[\mathcal{P}_{\mathcal{S}^{\prime}}\right]\right)}\left(\iota\left[\mathcal{P}_{\mathcal{S}^{\prime}}\right]\right) \cdot 15$

Of course, the choice of $\iota$ was only canonical up to permutation of the coordinates. However, permuting the coordinates does not affect the Hausdorff measure, let alone the Hausdorff dimension, in any way. Therefore, there is a profound sense in which the pair consisting of the Hausdorff dimension and Hausdorff measure of $\iota\left[\mathcal{P}_{\mathcal{S}}\right]$ is a natural, if not canonical way of measuring the size of $\mathcal{P}_{\mathcal{S}}$ - and hence, on a semantic understanding of probabilistic consistency, the degree of probabilistic consistency of $\mathcal{S}$.

However, while it is possible to define a graded measure of probabilistic consistency, it is much less clear what the relation of the graded notion of probabilistic consistency to coherence is: If a belief

\footnotetext{
${ }^{15}$ In other words, if the values of $\tilde{\beta}_{2}$ are used to measure probabilistic consistency, they should to be read in lexicographic order, so that the first component of $\tilde{\beta}_{2}$ always trumps the second one.
} 
system is so acute that only a single probability measure is compatible with it, should we view this as a token for coherence - or rather for incoherence, because it borders probabilistic inconsistency by a knife's edge? Our intuitions seem to point in both directions at once, perhaps with a greater weight on the latter (incoherence). However, all that this shows is that the relation of graded probabilistic consistency and coherence is not yet sufficiently understood to warrant the inclusion of graded probabilistic consistency into our vector-valued coherence measure.

Therefore, we stick with the above definition of $\beta_{2}$ derived from the binary notion of probabilistic consistency. We do not propose the graded notion of probabilistic consistency described above, despite being of interest in its own right, to enter the graded coherence notion defined in this paper.

5.5. Formalising BonJour's third and fourth desiderata. For BonJour's third and fourth requirements, we suggest viewing a belief system $\mathcal{S}$ as a directed graph, ${ }^{16}$ such that the vertices (nodes) are propositions to which any agent with belief system $\mathcal{S}$ assigns a sufficiently high degree of belief and such that an arrow from $B$ to $A$ means that $B$ confirms $A$ (in the sense of Bayesian confirmation theory) with respect to the belief system $\mathcal{S}$.

On the one hand, such a representation of belief systems would neatly formalise coherentist intuitions such as Quine's and Ullian's “web of belief" 61. On the other hand, it is plagued by the invocation of the concept of belief simpliciter (the vertices are supposed to be propositions which the agent believes tout court) within a framework among whose basic concepts is that of a degree of belief.

We propose to solve this by means of a set of directed graphs. First, there is what we shall extended web of belief $H_{\S}$. Here, the vertices are all those propositions $A \in \mathcal{A}$ that are at least candidates for objects of full belief in the sense that $P(A)>1 / 2$ for all $P \in \mathcal{P}_{\mathcal{S}}$. There will be an arrow between vertex $B$ and vertex $A$ if and only if $B$ confirms $A$ in the sense of Bayesian confirmation theory (with the belief system $\mathcal{S}$ in the background), i.e. if and only if $P(A \mid B)-P(A)>0$ for all $P \in \mathcal{P}_{\mathcal{S}}$. The extended web of belief contains propositions as vertices to which not a precise probability, but merely a lower bound is assigned by the belief system (e.g. all events/propositions that extensionally dominate an event/proposition to which $\mathcal{S}$ unconditionally assigns some precise probability $>1 / 2$, cf. footnote 7 ).

Then there is the inner web of belief $G_{\text {s. }}$. Here, the vertices are all those propositions $A \in \mathcal{A}$ which might be verified to be objects of full belief for suitable thresholds $c$ in the Lockean thesis. More precisely, the vertices of $G_{\mathcal{S}}$ are all those propositions $A \in \mathcal{A}$ to which precise

\footnotetext{
${ }^{16}$ The graph-theoretic terminology is taken from Diestel $\mathbf{1 8}$.
} 
unconditional probabilities $>1 / 2$ are assigned by the belief system, in the sense that there is a real number $\alpha>1 / 2$ such that $P(A)=\alpha$ for all $P \in \mathcal{P}_{\mathcal{S}}$. There will be an arrow between vertex $B$ and vertex $A$ if and only if $B$ confirms $A$ in the sense of Bayesian confirmation theory (with the belief system $\mathcal{S}$ in the background) with a precise degree of confirmation, i.e. if and only if there exists some real number $\gamma>0$ such that $P(A \mid B)-P(A)=\gamma$ for all $P \in \mathcal{P}_{\mathcal{S}}$. For any such $A, B$, we shall refer to this positive real $\gamma$ as $\gamma(B, A)$. For those $B, A$, for which there is no $\gamma>0$ that would satisfy $P(A \mid B)-P(A)=\gamma$ for all $P \in \mathcal{P}_{\mathcal{S}}$ exists, we simply put $\gamma(B, A)=0$.

In addition, one could define, for any $c \geq 1 / 2$, the $c$-core of beliefs as the subgraph $G_{\S}^{c}$ of $G_{\mathcal{S}}$ which consists of only those propositions $A$ to which precise unconditional probabilities $>c$ are assigned by the belief system, in the sense that there is a real number $\alpha>c$ such that $P(A)=\alpha$ for all $P \in \mathcal{P}_{\mathcal{S}}$. For all $c \geq 1 / 2, G_{\mathcal{S}}^{c}$ as well as $G_{\mathcal{S}}$ and $H_{\mathcal{S}}$ are Bayesian networks.

Now there are two aspects to BonJour's requirement (III), the first being that "[t]he coherence of a system of beliefs is increased by the presence of inferential connections between its component beliefs and increased in proportion to the number [...] of such connections". We suggest capturing this by the graph-theoretic notion of (vertex) connectivity $\kappa$. In light of the adaptation of Menger's theorem [53] (cf. also Whitney 73 and König [42]) to directed graphs (cf. Gallai 30 and Dirac [20, 21]), this notion can even be defined in two equivalent natural ways, one of them being that the connectivity $\kappa(G)$ of a graph $G$ equals $k$ if and only if for any two vertices $A, B$, there are at least $k$ independent paths from $A$ to $B{ }^{17}$ but there are also two vertices $A^{\prime}, B^{\prime}$ such that one cannot find $k+1$ independent paths from $A^{\prime}$ to $B^{\prime}$.

The second of the two aspects of BonJour's requirement (III) demands that "[t]he coherence of a system of beliefs is increased [...] in proportion to the [...] strength of [inferential] connections [between its component beliefs]". Of course, in a Bayesian setting, the most natural interpretation of the "strength of an inferential connection" from $B$ to $A$ is the degree by which $B$ confirms $A$ (in the sense of Bayesian confirmation theory). While there are several quantitative measures for the degree of confirmation in Bayesian confirmation theory, the (by far) most widely-used among them is the relevance measure, also known as the difference measure of confirmation, which defines the degree by which $B$ confirms $A$ as $P(A \mid B)-P(A)$. Another, more direct, motivation for using the relevance measure to assess coherence is the following: If a set of beliefs really coheres (hangs together well), it should be possible to repair local loss of information. Thus, there will in general be multiple pieces of confirmatory evidence for assigning a high

\footnotetext{
${ }^{17} \mathrm{~A}$ collection of paths is independent if and only if none of the paths in that collection contains an inner vertex of another path from that collection.
} 
degree of belief in a certain proposition, and even in the absence of one of those pieces of evidence, there will be other pieces of confirmatory evidence which warrant the assignment of a lower, but still considerable degree of belief to that proposition.

Thus, we propose to measure the number and strength of initial connections in the belief system by vertex connectivity and the vector of positive relevance values:

$$
\beta_{3}(\mathcal{S}):=\left\langle\kappa\left(G_{\mathcal{S}}\right),\langle\gamma(B, A): A, B \in \mathcal{A}\rangle\right\rangle .
$$

The penultimate requirement of BonJour's is (IV), which demands that the relative fragmentation of a belief system (given the overall level of connectivity within the belief system) diminish coherence. This can be accommodated by defining $\beta_{4}(\mathcal{S})$ to be (i) the reciprocal cardinality of the set of maximal $\kappa\left(G_{\delta}\right)+1$-connected proper subgraphs, i.e. $\kappa\left(G_{\mathcal{S}}\right)+1$-components, if this set is non-empty and (ii) zero otherwise. For example, in the special case when $\kappa\left(G_{\mathcal{S}}\right)=0$ (i.e. $G_{\mathcal{S}}$ is totally disconnected), we have $\beta_{4}(\mathcal{S})=0$.

5.6. BonJour's fifth desideratum. It is not nearly as clear how to formalise the fifth and last requirement - i.e. the negative "influence of unexplained anomalies" on the degree of coherence be it in our formal framework or in another. That said, BonJour considered (V) to be only an "advisable" [6, p. 99] and thus perhaps not mandatory ingredient of a coherence theory of epistemic justification. At least for the first four (essential) criteria, we have proposed rigorous formalisations.

5.7. Taking stock of the formalisation. Generalisation to infinite belief systems. In sum, we have proposed a vector-valued measure of coherence $\gamma$, which assigns to a belief system $\mathcal{S}$ the vector

$$
\gamma(\mathcal{S})=\left\langle\beta_{1}(\mathcal{S}), \beta_{2}(\mathcal{S}), \beta_{3}(\mathcal{S}), \beta_{4}(\mathcal{S})\right\rangle,
$$

with $\beta_{1}, \beta_{2}, \beta_{3}, \beta_{4}$ defined as above, and thus formalises what may be called the thrust of BonJour's coherence concept.

Note that this definition of a vector-valued coherence measure only applies, as it stands, to finite belief systems, whilst coherentists are generally committed to a holistic approach that takes into account the whole worldview of an agent, which may well be an infinite belief system. In order to obtain a vector-valued coherence measure which is defined for some infinite belief systems, one can simply resort to Robinsonian [63, 64] nonstandard analysis: By the transfer principle of nonstandard analysis, our vector-valued coherence measure will then not only be defined for all finite belief systems, but even for all hyperfinite belief systems.

However, it can be shown (cf. Fajardo and Keisler [24]) that hyperfinite probability spaces are universal and saturated in a rigorous model-theoretic sense (based on a model theory of random variables 
and stochastic processes). Therefore, we may without loss of generality assume that any infinite belief system has been defined on a hyperfinite probability space from the start. The range of the coherence measure will then be a set of vectors of hyperreals - but nothing in the concept of coherence measure requires its range to consist of reals or tuples of reals only.

\section{Examples. BonJour's challenge}

\subsection{Preparations for the formalisation of BonJour's} challenge. We shall now illustrate our coherence definition by means of some examples. First, let us recall BonJour's [6, p. 96] challenge, which has been neatly summarised by Bovens and Hartmann [9, p. 618]. Consider the following propositions:

$\tilde{R}_{1}$ : 'All ravens are black.'

$R_{2}$ : 'This bird is a raven.'

$R_{3}$ : 'This bird is black.'

$R_{1}^{\prime}$ : 'This chair is brown.'

$R_{2}^{\prime}$ : 'Electrons are negatively charged.'

$R_{3}^{\prime}$ : 'Today is Thursday.'

Let $\tilde{\mathcal{R}}=\left\{\tilde{R}_{1}, R_{2}, R_{3}\right\}$ and $\mathcal{R}^{\prime}=\left\{R_{1}^{\prime}, R_{2}^{\prime}, R_{3}^{\prime}\right\}$. In Bovens' and Hartmann's words, "The challenge is to give an account of the fact that $[\tilde{\mathcal{R}}]$ is more coherent than $\left[\mathcal{R}^{\prime}\right] . "$ [9, p. 618]

Of course, it is tacitly assumed herein that among $R_{1}^{\prime}, R_{2}^{\prime}, R_{3}^{\prime}$, no inferential connections are known (to the individual who holds this belief system). The belief systems that are (in approximation) represented by the information sets $\mathcal{R}$ and $\mathcal{R}^{\prime}$ should be understood to embody no inferential connections except for the obvious one, viz. modus ponens inference from $R_{1}, R_{2}$ to $R_{3}$.

Moreover, in contrast to the other propositions in $\tilde{\mathcal{R}} \cup \mathcal{R}^{\prime}$, the proposition $\tilde{R}_{1}$ can be regarded as a scheme of inferential connections rather than as a proposition (and therefore has been typographically set apart). In the context of the belief system represented by $\tilde{\mathcal{R}}$, it could also be replaced by a mere single inferential connection such as:

$R_{3} \mid R_{2}$ : 'If this bird is a raven, then it is black.'

Indeed, if one wants to take a thoroughly Bayesian approach to belief systems, the most natural way forward is to formalise belief systems as Bayesian networks, as we have done in the preceding sections. However, incorporating a scheme of inferential connections into a Bayesian network amounts to incorporating each of the instantiations as inferential connections into the Bayesian network. In the context of our example, the scheme $\tilde{R}_{1}$ only implies one such inferential connection, viz. $R_{3} \mid R_{2}$. 
We shall henceforth study $\mathcal{R}:=\left\{R_{3} \mid R_{2}, R_{2}, R_{3}\right\}$ en lieu of $\tilde{\mathcal{R}}$. BonJour's challenge is then to give an account of the greater coherence of $\mathcal{R}$ compared to that of $\mathcal{R}^{\prime}$.

6.2. Formalising the belief systems involved in BonJour's challenge. In order to meet that challenge by applying the formal analysis of the preceding sections, we first need to translate $\mathcal{R}$ and $\mathcal{R}^{\prime}$ into belief systems $\mathcal{S}, \mathcal{S}^{\prime}$ as we (from our rigorously Bayesian perspective) understand them.

In our setting, if the information available to a rational individual is — in approximation — represented by $\mathcal{R}$ (or $\mathcal{R}^{\prime}$, respectively), this means something like:

(1) she assigns to each of the propositions/events and conditional events in $\mathcal{R}$ (or $\mathcal{R}^{\prime}$, respectively) a sufficiently high degree of belief;

(2) all degrees of belief that she assigns to other conditional events assume pairwise independence of the propositions constituting the conditional event in question.

The first step is the choice of an algebra of propositions/events to which conditional degrees of belief will be assigned. Since there are only finitely many propositions involved, we may model each situation on the basis of a finite algebra $\mathcal{A}$ or $\mathcal{A}^{\prime}$ - which, in turn we may identify with power-set algebras $2^{\Omega}$ and $2^{\Omega^{\prime}}$, respectively. Applied to our choices of $\mathcal{R}$ and $\mathcal{R}^{\prime}$, the above translation program yields the following.

- An individual with information set $\mathcal{R}$ has a belief system $\mathcal{S}$ consisting of:

- $\left\langle R_{i} \mid \Omega \| \alpha_{i}\right\rangle$ for each $i \in\{2,3\}$ for some $\alpha_{i} \in[0,1]$ that is sufficiently close to 1 ;

- $\left\langle R_{3} \mid R_{2} \| \alpha_{1}\right\rangle$ for some $\alpha_{1} \in[0,1]$ that is sufficiently close to 1 and strictly greater than $\alpha_{3}$.

Therefore, for the belief system $\mathcal{S}$, the set $\Omega$ of states of the world only needs four elements, given by the state descriptions ' $R_{2}$ and $R_{3}$ ', ' $R_{2}$ but not $R_{3}$ ', ' $R_{3}$ but not $R_{3}$ ', 'neither $R_{2}$ not $R_{3}$ '. More formally, let us define the states of the world as follows:

$$
\begin{array}{cc}
\omega^{(1)}:=\left\langle\dot{R}_{2}, \dot{R}_{3}\right\rangle & \omega^{(2)}:=\left\langle\dot{R}_{2}, \dot{\neg} \dot{R}_{3}\right\rangle \\
\omega^{(3)}:=\left\langle\dot{\neg} \dot{R}_{2}, \dot{R}_{3}\right\rangle & \omega^{(4)}:=\left\langle\dot{\neg} \dot{R}_{2}, \dot{\neg} \dot{R}_{3}\right\rangle .
\end{array}
$$

(Herein, $\neg$ denotes propositional negation, and the dots indicate that $\dot{R}_{i}$ is a mere name for the proposition $R_{i}$, but not the proposition itself - which, when identified with extension, is a set of states of the world!)

- An individual with information set $\mathcal{R}^{\prime}$ has a belief system $\mathcal{S}^{\prime}$ consisting of: 
- $\left\langle R_{1}^{\prime} \mid \Omega^{\prime} \| \alpha_{1}^{\prime}\right\rangle,\left\langle R_{1}^{\prime} \mid R_{2}^{\prime} \| \alpha_{1}^{\prime}\right\rangle,\left\langle R_{1}^{\prime} \mid R_{3}^{\prime} \| \alpha_{1}^{\prime}\right\rangle$ for some $\alpha_{1}^{\prime} \in[0,1]$

- $\left\langle R_{2}^{\prime} \mid \Omega^{\prime} \| \alpha_{2}^{\prime}\right\rangle,\left\langle R_{2}^{\prime} \mid R_{1}^{\prime} \| \alpha_{2}^{\prime}\right\rangle,\left\langle R_{2}^{\prime} \mid R_{3}^{\prime} \| \alpha_{2}^{\prime}\right\rangle$ for some $\alpha_{2}^{\prime} \in[0,1]$

$-\left\langle R_{3}^{\prime} \mid \Omega^{\prime} \| \alpha_{3}^{\prime}\right\rangle,\left\langle R_{3}^{\prime} \mid R_{1}^{\prime} \| \alpha_{3}^{\prime}\right\rangle,\left\langle R_{3}^{\prime} \mid R_{2}^{\prime} \| \alpha_{3}^{\prime}\right\rangle$ for some $\alpha_{3}^{\prime} \in[0,1]$, wherein for every $i \in\{1,2,3\}$, $\alpha_{i}^{\prime}$ is sufficiently close to 1 . Therefore, for the belief system $\mathcal{S}^{\prime}$, the set $\Omega^{\prime}$ of states of the world only needs eight elements:

$$
\begin{aligned}
\omega^{\prime(1)}:=\left\langle\dot{R}_{1}^{\prime}, \dot{R}_{2}^{\prime}, \dot{R}_{3}^{\prime}\right\rangle & \omega^{(2)}:=\left\langle\dot{R}_{1}^{\prime}, \dot{R}_{2}^{\prime}, \dot{\neg} \dot{R}_{3}^{\prime}\right\rangle \\
\omega^{\prime(3)}:=\left\langle\dot{R}_{1}^{\prime}, \dot{\neg} \dot{R}_{2}^{\prime}, \dot{R}_{3}^{\prime}\right\rangle & \omega^{(4)}:=\left\langle\dot{R}_{1}^{\prime}, \dot{\neg} \dot{R}_{2}^{\prime}, \dot{\neg} \dot{R}_{3}^{\prime}\right\rangle \\
\omega^{\prime(5)}:=\left\langle\dot{\neg} \dot{R}_{1}^{\prime}, \dot{R}_{2}^{\prime}, \dot{R}_{3}^{\prime}\right\rangle & \omega^{\prime(6)}:=\left\langle\dot{\neg} \dot{R}_{1}^{\prime}, \dot{R}_{2}^{\prime}, \dot{\neg} \dot{R}_{3}^{\prime}\right\rangle \\
\omega^{\prime(7)}:=\left\langle\dot{\neg} \dot{R}_{1}^{\prime}, \dot{\neg} \dot{R}_{2}^{\prime}, \dot{R}_{3}^{\prime}\right\rangle & \omega^{\prime(8)}:=\left\langle\dot{\neg} \dot{R}_{1}^{\prime}, \dot{\neg} \dot{R}_{2}^{\prime}, \dot{\neg} \dot{R}_{3}^{\prime}\right\rangle
\end{aligned}
$$

We now need to compute - or at least estimate - the several components $\beta_{1}, \beta_{2}, \beta_{3}, \beta_{4}$ of our coherence measure applied to $\mathcal{S}$ and $\mathcal{S}^{\prime}$. We need to show that according to our multi-dimensional coherence measure, the coherence of $\mathcal{S}$ dominates that of $\mathcal{S}^{\prime}$ - except perhaps in extraordinary circumstances.

For the application of our coherence measure (in particular for $\beta_{3}, \beta_{4}$ ), we need to represent $\mathcal{S}$ and $\mathcal{S}^{\prime}$ as Bayesian networks. The belief system $\mathcal{S}^{\prime}$ is represented by a completely disconnected graph $G_{\mathcal{S}^{\prime}}$ with three vertices, so that

$$
\beta_{3}\left(\mathcal{S}^{\prime}\right)=\langle 0,\langle 0, \ldots, 0\rangle\rangle, \quad \beta_{4}\left(\mathcal{S}^{\prime}\right)=0 .
$$

The belief system $\mathcal{S}$ consists of two vertices with an arrow from $R_{2}$ ('This bird is a raven') to $R_{3}$ ('This bird is black').

We shall now calculates $\beta_{1}, \beta_{2}, \beta_{3}, \beta_{4}$ for $\mathcal{S}$ and $\mathcal{S}^{\prime}$; we shall also estimate $\tilde{\beta}_{2}$ for both belief systems. For reasons of economy of presentation, we start with $\beta_{3}, \beta_{4}$.

6.3. Calculation of the third and fourth component of the coherence measure. Given the above descriptions of the Bayesian networks $G_{\mathcal{S}}, G_{\mathcal{S}^{\prime}}$ associated to the belief systems $\mathcal{S}, \mathcal{S}^{\prime}$, the (vertex) connectivity of $G_{\mathcal{S}}$ is 1 , the single inferential non-zero connection in $G_{\mathcal{S}}$ being the one from $R_{2}$ to $R_{3}$, whose strength is $\gamma\left(R_{2}, R_{3}\right)=\alpha_{1}-\alpha_{3}>0$.

In contrast, the (vertex) connectivity of $G_{\mathcal{S}^{\prime}}$ is 0 , and thus the vector of strengths of inferential connections among the vertices in $G_{\mathcal{S}^{\prime}}$ is trivial (consists of zeroes only). Thus,

$$
\begin{aligned}
& \beta_{3}(\mathcal{S})=\left\langle 1,\langle\underbrace{\alpha_{1}-\alpha_{3}}_{>0}, 0, \ldots, 0\rangle\right\rangle . \\
& \beta_{3}\left(\mathcal{S}^{\prime}\right)=\langle 0,\langle 0, \ldots, 0\rangle\rangle .
\end{aligned}
$$

The relative fragmentation of both $\mathcal{S}$ and $\mathcal{S}^{\prime}$ is zero: $G_{\mathcal{S}}$ has connectivity 1 , but no 2-connected components; $G_{\mathcal{S}^{\prime}}$ has connectivity 
0 (is totally disconnected) and therefore cannot have any 1-connected components. Thus,

$$
\beta_{4}(\mathcal{S})=0=\beta_{4}\left(\mathcal{S}^{\prime}\right) .
$$

6.4. Calculation of graded probabilistic consistency. The second component of the coherence measure. We shall calculate $\tilde{\beta}_{2}$ for $\mathcal{S}, \mathcal{S}^{\prime}$; as a by-product, this will yield probabilistic consistency proofs for both $\mathcal{S}$ and $\mathcal{S}^{\prime}$. Recall that $\tilde{\beta}_{2}$ was defined as the size of the set $\mathcal{P}$ of probability measures compatible with the belief system in question: We made the - arguably rather natural - proposal of measuring the size of $\mathcal{P}$ in terms of the Hausdorff dimension and Hausdorff measure of the canonical geometrical representation $\iota[\mathcal{P}]$ of $\mathcal{P}$ as an intersection of hyperplanes with the set of probability measures (on a finite set whose power-set is isomorphic to the algebra of propositions with respect to which beliefs are formed), which itself is the intersection of a hyperplane with a unit cube.

Now, in the case of $\mathcal{S}$, the canonical geometrical representation $\iota\left[\mathcal{P}_{\mathcal{S}}\right]$ is the intersection of the four-dimensional unit cube with four hyperplanes, one for each of the three (conditional) probability assignment) plus the hyperplane generated by requiring the total mass to add up to one:

$$
\iota\left[\mathcal{P}_{\mathcal{S}}\right]=\left\{\begin{array}{cc} 
& x^{(1)}+x^{(2)}+x^{(3)}+x^{(4)}=1, \\
x \in[0,1]^{4}: & x^{(1)}=\left(x^{(1)}+x^{(2)}\right) \alpha_{1}, \\
& x^{(1)}+x^{(2)}=\alpha_{2}, \\
x^{(1)}+x^{(3)}=\alpha_{3} .
\end{array}\right\} .
$$

Herein, any $x=\left\langle x^{(1)}, x^{(2)}, x^{(3)}, x^{(4)}\right\rangle \in \iota\left[\mathcal{P}_{\mathcal{S}}\right]$ represents a probability measure on the power-set of $\Omega=\left\{\omega^{(1)}, \omega^{(2)}, \omega^{(3)}, \omega^{(4)}\right\}$ that is compatible with $\mathcal{S}$ and assigns probability $x^{(i)}$ to $\left\{\omega^{(i)}\right\}$ for each $i \in\{1,2,3,4\}$; the three (conditional) probability assignments that make up $\mathcal{S}$ have been encoded accordingly. ${ }^{18}$ By elementary linear algebra, this reduces to

$$
\iota\left[\mathcal{P}_{\mathcal{S}}\right]=\left\{\left(\begin{array}{c}
\alpha_{1} \alpha_{2} \\
\alpha_{2}\left(1-\alpha_{1}\right) \\
\alpha_{3}-\alpha_{1} \alpha_{2} \\
1-\alpha_{2}\left(1-\alpha_{1}\right)-\alpha_{3}
\end{array}\right)\right\}
$$

\footnotetext{
${ }^{18}$ For example, the assignment of a probability of $\alpha_{1}$ to the conditional event $R_{3} \mid R_{2}$ requires that the probability of $R_{2} \cap R_{3}$ equals $\alpha_{1}$ times the probability of $R_{2}$. The former proposition corresponds to $\left\{\left\langle\dot{R}_{2}, \dot{R}_{3}\right\rangle\right\}=\left\{\omega^{(1)}\right\}$, the latter proposition to $\left\{\left\langle\dot{R}_{2}, \dot{R}_{3}\right\rangle,\left\langle\dot{R}_{2}, \dot{\neg} \dot{R}_{3}\right\rangle\right\}=\left\{\omega^{(1)}, \omega^{(2)}\right\}$. Therefore, the conditional subjective probability assignment $\left\langle R_{3} \mid R_{2} \| \alpha_{1}\right\rangle$ from $\mathcal{S}$ corresponds to the equation $x^{(1)}=\left(x^{(1)}+x^{(2)}\right) \alpha_{1}$ in the above formula for $\iota\left[\mathcal{P}_{\mathcal{S}}\right]$. In a similar vein, the conditional subjective probability assignment $\left\langle R_{2} \mid \Omega \| \alpha_{2}\right\rangle$ from $\mathcal{S}$ corresponds to the equation $x^{(1)}+x^{(2)}=\alpha_{2}$ in the above formula for $\iota\left[\mathcal{P}_{\mathcal{S}}\right]$, and the degree of belief $\left\langle R_{3} \mid \Omega \| \alpha_{3}\right\rangle$ corresponds to the equation $x^{(1)}+x^{(3)}=\alpha_{3}$.
} 
This is a singleton and thus its Hausdorff dimension is zero and so is its Hausdorff measure: Therefore, according to our proposal in the above excursion, the degree of probabilistic consistency of $\mathcal{S}$ must be represented by two zeroes.

$$
\tilde{\beta}_{2}(\mathcal{S})=\langle 0,0\rangle
$$

This, however, does not mean that $\mathcal{S}$ is probabilistically inconsistent, just that is in a sense 'minimally consistent' probabilistically.

Rather, $\mathcal{P}_{\mathcal{S}}$ is a singleton (since $\iota\left[\mathcal{P}_{\mathcal{S}}\right]$ is a singleton) and thus in particular non-empty, so

$$
\beta_{2}(\mathcal{S})=1
$$

In other words, the belief system $\mathcal{S}$ is probabilistically consistent, and $\mathcal{S}$ induces a unique probability system that is compatible with $\mathcal{S}$.

For the probabilistic consistency component of BonJour's challenge, this is already sufficient: It shows that $\mathcal{S}$ cannot be dominated in terms of probabilistic consistency simpliciter by $\mathcal{S}^{\prime}$. Since we have already seen that $\mathcal{S}$ dominates $\mathcal{S}^{\prime}$ in other aspects of coherence, all we need to show that $\mathcal{S}$ fares at least as well as $\mathcal{S}^{\prime}$ in terms of logical consistency and probabilistic consistency. (In fact, BonJour [6, p. 95f.]introduced the belief systems that we modelled by $\mathcal{S}$ and $\mathcal{S}^{\prime}$ as an example of two belief systems which cannot be distinguished by logical or probabilistic consistency, but still cannot reasonably called equally coherent.)

Nevertheless, for the sake of illustration, we shall also compute $\tilde{\beta}_{2}\left(\mathcal{S}^{\prime}\right)$. A similar analysis for $\mathcal{S}^{\prime}$ will show that $\iota\left[\mathcal{P}_{\mathcal{S}^{\prime}}\right]$ is actually given by the following linear equation system:

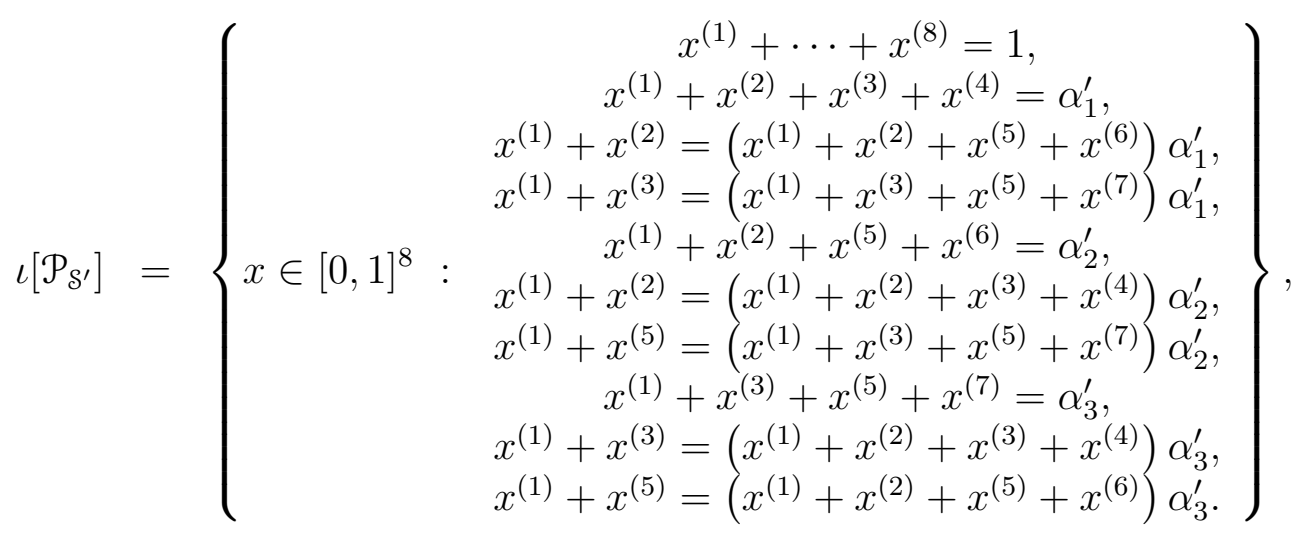


which can be simplified to become ${ }^{19}$

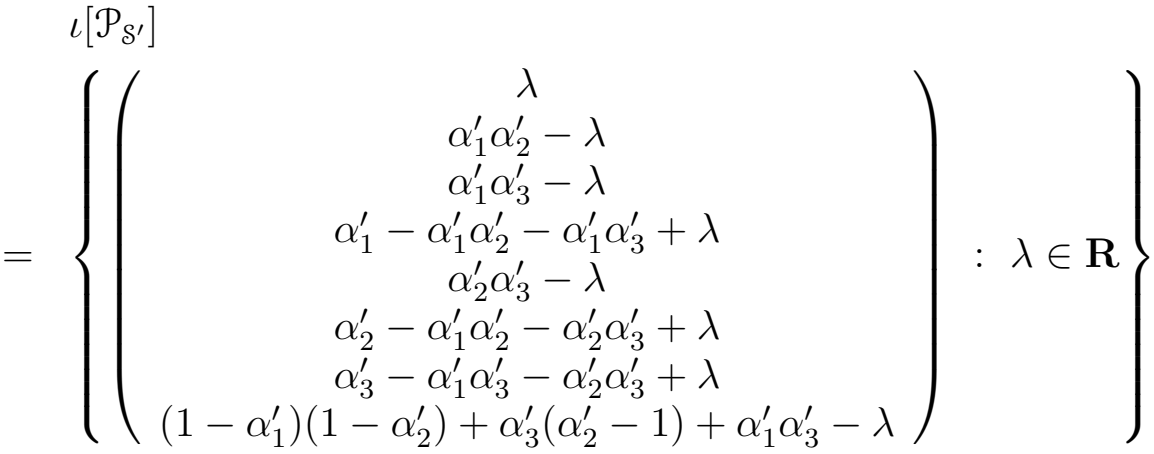

$$
\begin{aligned}
& \cap[0,1]^{8}
\end{aligned}
$$

${ }^{19}$ By elementary linear algebra, one first gets

$$
\iota\left[\mathcal{P}_{\mathcal{S}^{\prime}}\right]=\left\{\begin{array}{c}
x^{(1)}+\cdots+x^{(8)}=1, \\
x^{(1)}+x^{(2)}+x^{(3)}+x^{(4)}=\alpha_{1}^{\prime}, \\
x^{(1)}+x^{(2)}=\alpha_{1}^{\prime} \alpha_{2}^{\prime}, \\
x^{(1)}+x^{(3)}=\alpha_{1}^{\prime} \alpha_{3}^{\prime}, \\
x^{(1)}+x^{(2)}+x^{(5)}+x^{(6)}=\alpha_{2}^{\prime}, \\
x^{(1)}+x^{(2)}=\alpha_{1}^{\prime} \alpha_{2}^{\prime}, \\
x^{(1)}+x^{(5)}=\alpha_{2}^{\prime} \alpha_{3}^{\prime}, \\
x \in[0,1]^{8}: x^{(3)}+x^{(5)}+x^{(7)}=\alpha_{3}^{\prime}, \\
x^{(1)}+x^{(3)}=\alpha_{1}^{\prime} \alpha_{3}^{\prime}, \\
x^{(1)}+x^{(5)}=\alpha_{2}^{\prime} \alpha_{3}^{\prime} .
\end{array}\right\}
$$

which after dropping repeated equations reduces to

$$
\iota\left[\mathcal{P}_{\mathcal{S}^{\prime}}\right]=\left\{\begin{array}{c}
x^{(1)}+\cdots+x^{(8)}=1, \\
x^{(1)}+x^{(2)}+x^{(3)}+x^{(4)}=\alpha_{1}^{\prime}, \\
x^{(1)}+x^{(2)}=\alpha_{1}^{\prime} \alpha_{2}^{\prime}, \\
x^{(1)}+x^{(3)}=\alpha_{1}^{\prime} \alpha_{3}^{\prime}, \\
x \in[0,1]^{8}: \\
\\
x^{(1)}+x^{(2)}+x^{(5)}+x^{(6)}=\alpha_{2}^{\prime}, \\
x^{(1)}+x^{(3)}+x^{(5)}+x^{(7)}=\alpha_{3}^{\prime}, \\
x^{(1)}+x^{(5)}=\alpha_{2}^{\prime} \alpha_{3}^{\prime} .
\end{array}\right\}
$$

and finally, solving for $x^{(1)}$ and inserting the solutions,

$$
\iota\left[\mathcal{P}_{\mathcal{S}^{\prime}}\right]=\left\{\begin{array}{c}
x^{(2)}=\alpha_{1}^{\prime} \alpha_{2}^{\prime}-x^{(1)}, \\
x^{(3)}=\alpha_{1}^{\prime} \alpha_{3}^{\prime}-x^{(1)}, \\
x \in[0,1]^{8}: \quad \alpha_{1}^{\prime}-\alpha_{1}^{\prime} \alpha_{2}^{\prime}-\alpha_{1}^{\prime} \alpha_{3}^{\prime}+x^{(1)}, \\
\\
x^{(5)}=\alpha_{2}^{\prime} \alpha_{3}^{\prime}-x^{(1)}, \\
x^{(6)}=\alpha_{2}^{\prime}-\alpha_{1}^{\prime} \alpha_{2}^{\prime}-\alpha_{2}^{\prime} \alpha_{3}^{\prime}+x^{(1)}, \\
x^{(7)}=\alpha_{3}^{\prime}-\alpha_{1}^{\prime} \alpha_{3}^{\prime}-\alpha_{2}^{\prime} \alpha_{3}^{\prime}+x^{(1)}, \\
x^{(8)}=1-\alpha_{1}^{\prime}-\alpha_{2}^{\prime}+\alpha_{1}^{\prime} \alpha_{2}^{\prime}+\alpha_{2}^{\prime} \alpha_{3}^{\prime}-\alpha_{3}^{\prime}+\alpha_{1}^{\prime} \alpha_{3}^{\prime}-x^{(1)}
\end{array}\right\}
$$


In other words, $\iota\left[\mathcal{P}_{\mathcal{S}^{\prime}}\right]$ consists of all vectors of the eight-dimensional unit cube that can be written in the form

$$
\left(\begin{array}{c}
0 \\
\alpha_{1}^{\prime} \alpha_{2}^{\prime} \\
\alpha_{1}^{\prime} \alpha_{3}^{\prime} \\
\alpha_{1}^{\prime}\left(1-\alpha_{2}^{\prime}-\alpha_{3}^{\prime}\right) \\
\alpha_{2}^{\prime} \alpha_{3}^{\prime} \\
\alpha_{2}^{\prime}\left(1-\alpha_{1}^{\prime}-\alpha_{3}^{\prime}\right) \\
\alpha_{3}^{\prime}\left(1-\alpha_{1}^{\prime}-\alpha_{2}^{\prime}\right) \\
\left(1-\alpha_{1}^{\prime}\right)\left(1-\alpha_{2}^{\prime}\right)+\alpha_{3}^{\prime}\left(\alpha_{2}^{\prime}-1\right)+\alpha_{1}^{\prime} \alpha_{3}^{\prime}
\end{array}\right)+\left(\begin{array}{c}
1 \\
-1 \\
-1 \\
1 \\
-1 \\
1 \\
1 \\
-1
\end{array}\right) \lambda
$$

for some real number $\lambda$. Clearly, the Hausdorff dimension of $\iota\left[\mathcal{P}_{\mathcal{S}^{\prime}}\right]$ is one, and its Hausdorff measure is positive.

6.5. Calculation of the first component of the coherence measure. The first component of our coherence measure, $\beta_{1}$, captures logical consistency. It is obvious from our above calculations of representations of $\mathcal{P}_{\mathcal{S}}$ and $\mathcal{P}_{\mathcal{S}^{\prime}}$ (under $\iota$ ) that only the top element of the Boolean algebra $\left(\Omega\right.$ in the case of belief system $\mathcal{S}$ on algebra $\mathcal{A} ; \Omega^{\prime}$ in the case of belief system $\mathcal{S}^{\prime}$ on algebra $\mathcal{A}^{\prime}$ ) is assigned probability 1 by every probability measure that is compatible with the respective belief system (be it $\mathcal{S}$ or $\left.\mathcal{S}^{\prime}\right)$. Therefore, $\eta(\mathcal{S},\{1\})=\Omega$ and $\eta\left(\mathcal{S}^{\prime},\{1\}\right)=\Omega^{\prime}$, whence

$$
\beta_{1}(\mathcal{S})=1=\beta_{1}\left(\mathcal{S}^{\prime}\right) .
$$

Just as claimed by BonJour when introducing the challenge [6, p. 95f.], $\mathcal{S}$ and $\mathcal{S}^{\prime}$ cannot be told apart by considering logical and probabilistic consistency (i.e. $\beta_{1}$ and $\beta_{2}$ ) alone.

6.6. Summary. As we have seen,

$$
\begin{array}{ll}
\beta_{1}(\mathcal{S})=\beta_{1}\left(\mathcal{S}^{\prime}\right), & \beta_{2}(\mathcal{S})=\beta_{2}\left(\mathcal{S}^{\prime}\right), \\
\beta_{3}(\mathcal{S})>\beta_{3}\left(\mathcal{S}^{\prime}\right), & \beta_{4}(\mathcal{S})=\beta_{4}\left(\mathcal{S}^{\prime}\right) .
\end{array}
$$

The whole point of BonJour's [6, p. 95f.] giving the example of these two belief systems was that they are indistinguishable in terms of logical and probabilistic consistency, but the former is more coherent than the latter on account of inferential connections. This is exactly what the first three relations in the above formula array (4) teach us. Thus, our formal coherence notion has passed the test; it does live up to BonJour's challenge.

So far, we have merely constructed one coherence measure for each of BonJour's first four desiderata. We doubt that much more can be achieved without specifying the exact epistemic context. The epistemic context may, however, provide additional information on how to balance the four components of our vector-valued coherence measure (perhaps even in a non-linear way). Taking this into account, one can take the weighted average of the components (perhaps after 
applying a suitable monotone transformation in order to reflect any non-linearities).

\section{Discussion}

7.1. Summary of this paper. In this paper, we have proposed a formal framework for the analysis of Bayesian belief systems and, within that framework, a formal definition for the (vector-valued) degree of coherence of belief systems. Our definition is a formalisation of the essential parts of BonJour's [6] coherence concept, and it passes the test of BonJour's "ravens" challenge. It can be used as a point of departure to construct single-valued coherences measures satisfying BonJour's desiderata, e.g. by forming a weighted average (with weights depending on the epistemic context) of its components.

\subsection{Reply to objections against vector-valued coherence} measures. There is an obvious objection to a vector-valued coherence measure: The range of our coherence measure does not consist of (hyper)reals, but in general of hyperfinite sequences of hyperreals. Now there is, of course, more than one natural linear order on Cartesian powers of the (hyper)reals. Therefore, the set of coherence values cannot be linearly ordered in a non-arbitrary way, whence the coherence of belief systems will generally be incomparable.

On the one hand, this may be a disappointment for coherentists who had hoped for a single-valued coherence measure. On the other hand, given the multi-faceted nature of coherence, it is hardly surprising that a formal coherence notion should be a multi-dimensional concept.

In fact, there may be epistemic contexts where it is quite clear how to order the set of coherence values - e.g. by defining a (hyper)realvalued function, increasing in each component and of low degree of complexity, on the set of coherence values - and thus to obtain a scalar, single-dimensional coherence measure for that context. This does not contradict Bovens and Hartmann [11] who showed the impossibility of coherence rankings satisfying certain desiderata, as will be explained below in Subsection 7.3 .

One can also give the following reply: On the basis of our vectorvalued coherence measure, it is easily possible to find single-valued coherences measures that satisfy the first four desiderata laid down by BonJour. The reason is that those desiderata only demand that features of a doxastic system should ceteris paribus positively (or in some instances negatively) affect coherence. Therefore, the composition of our vector-valued, say $\mathbf{R}^{N}$-valued, coherence measure with any componentwise increasing function from $\mathbf{R}^{N}$ to $\mathbf{R}$ (for example, weighted averaging) yields a single-valued coherence measure satisfying the first four of BonJour's desiderata. 
7.3. Reply to objections citing impossibility theorems about coherence measures. In response to our above thesis about the feasibility of constructing a single-valued coherence measure from our vector-valued coherence measure, someone might invoke wellknown impossibility theorems, as for instance the one by Bovens and Hartmann [11. The reason for the compatibility of our possibility result with Bovens' and Hartmann's [11] impossibility result (and, for that matter, all other impossibility results in the literature reviewed above; they all work with a similar framework) is that the latter operate in a very different framework from ours, even conceptionally: $(\alpha)$ Their framework has, in contrast to ours, no room for partial belief; in the framework of Bovens' and Hartmann's [11 paper (as in all the existing literature on coherence measures reviewed above), the only intermediate state between believing a proposition and believing its negation is to have no doxastic attitude towards that proposition at all. $(\beta)$ Whosoever evaluates the coherence measure has a unique probability measure defined on the algebra generated by all propositions occurring in the doxastic states whose coherence is to be measured. $(\gamma)$ Doxastic states are, very much unlike in our framework, characterized by sets of propositions to which agents take a binary attitude. More precisely, while Bovens and Hartmann [11] conceive of doxastic states as sets of propositions, the present paper construes belief systems as collections of conditional probability assignments or, equivalently, as sets of probability measures compatible with such assignments (see Section 2). These differences imply that the domain of a coherence ranking in the sense of Bovens and Hartmann [11] is utterly different from the domain of our proposed graded coherence notion.

As a result, Bovens' and Hartmann's [11 desideratum called "Probabilism", while perfectly suitable to the existing formal literature on coherence measures, is not applicable to our framework. In addition, Bovens' and Hartmann's [11] desideratum of "Holism" the requirement that a transitive and complete ordering be defined on all non-empty information sets - is again very sensitive to how information sets are conceived, and need not be upheld when a merely local, context-specific coherence norm is sought.

7.4. Further objections. There are also, of course, potential objections to our formalisation of (the thrust of) BonJour's [6] coherence notion that relate to more general philosophical positions that the present paper subscribes to ${ }^{20}$

\footnotetext{
${ }^{20}$ Very recently, Hájek 31 has formulated (i) a powerful challenge to a core tenet of Bayesianism, viz. regularity, and (ii) D.W. Miller has offered a vigorous critique of Bayesian confirmation theory on account of its use of the relevance measure of confirmation (partly unpublished, but summarised in [54 and building upon the joint paper by Popper and Miller [60]). These are, of course, serious
} 
7.5. The dialectics of weak coherentism and its alternatives. So far, our discussion has concentrated on potential objections to our proposal of a formal coherence concept and their refutations. But one could also strike a less defensive note: In a recent paper (Herzberg [38]), a weakness of stronger versions of both foundationalism and infinitism that arises from the Principle of Inferential Justification (cf. e.g. Fumerton [28]) in a Bayesian setting is explored. This provides support for epistemological theories that do not assume a linear inferential structure of reasons, but instead adopt a holistic approach — which, of course, demands a concept of coherence that is both (a) formally defensible and (b) may serve the purposes of traditional epistemology. The present paper is an attempt at defining such a concept.

Adopting a coherence theory of epistemic justification along the lines sketched in this paper, however, does not mean that there cannot be foundational beliefs (as for instance those that Wittgenstein had in mind in his On certainty [74])! Our formal account explicitly allows for belief systems that include the assignment of an unconditional degreeof-belief of 1 to certain propositions; and such foundational beliefs in turn may entail, with a high conditional degree-of-belief, other beliefs, thus giving rise to a linear chain of reasons. However, unlike strong foundationalism, we do not suppose that every justified belief will have to belong to such a finite chain of reasons terminating in a foundational belief. In that sense, our version of coherentism is more liberal than strong versions of foundationalism.

7.6. Explanation and truth-conduciveness in coherentism. Since our graded coherence notion is a formalisation of the thrust of BonJour's [6] coherence concept, which exhibits some concern with explanatory relations (as the desiderata (III)-(V) reveal), it is

challenges to the overall project of a revived Bayesian coherentism, but a rebuttal would be way beyond its scope. We shall respond to both challenges in two forthcoming papers.

Another complaint against our graded coherence notion might be (iii) that our proposed formalisation could assign a non-minimal degree of coherence even to belief systems $\mathcal{S}$ that encode infinite regresses of probabilistic justification, provided $\mathcal{P}_{\mathcal{S}}$ is sufficiently large. In response, we point to a recent result by Herzberg [36, 37 that gives a criterion for the probabilistic consistency simpliciter of certain probabilistic regresses of epistemic justification which in turn can be used to refute influential arguments against regresses of epistemic justification.

Moreover, a critic might argue (iv) that our use of Robinsonian nonstandard analysis should subject us to a methodological criticism, since nonstandard analysis is allegedly fundamentally non-constructive (not just in a technical intuitionist sense). This objection can relatively easily be countered by referring to the metamathematical justification of nonstandard analysis (in terms of definable nonstandard models of the reals and entire nonstandard enlargements) contained in Kanovei and Shelah [41, Kanovei and Reeken [40], and Herzberg [34, 35. 
a suitable formal device for those coherence theories of epistemic justification that see coherence as a token of explanatory strength. Our coherence notion also passes the test of BonJour's ravens example, which can be seen as a test of the truth-conduciveness of a given coherence notion as much as of its explanatory significance.

7.7. A synthesis of BonJourian views. Since our coherence notion is graded it is well-suited to give a coherentist account of graded epistemic justification. It seems that such an account is consistent with a very narrow, Cartesian view of knowledge and full epistemic justification, as espoused in the more recent writings of Laurence BonJour [7 — for the simple reason that the latter position does not pose normative constraints on partial epistemic justification. ${ }^{21}$

\section{References}

[1] K. Akiba. Shogenji's probabilistic measure of coherence is incoherent. Analysis, 60(4):356-359, 2000.

[2] C.E. Alchourrón, P. Gärdenfors, and D. Makinson. On the logic of theory change: Partial meet contraction and revision functions. Journal of Symbolic Logic, 50(2):510-530, 1985.

[3] H. Arló-Costa and R. Parikh. Conditional probability and defeasible inference. Journal of Philosophical Logic, 34(1):97-119, 2005.

[4] H. Arló-Costa and A.P. Pedersen. Belief and probability: A general theory of probability cores. International Journal of Approximate Reasoning, 53(3):293$315,2012$.

[5] R. Audi. Epistemology: A contemporary introduction to the theory of knowledge. Routledge, Oxford, 3 edition, 2011.

[6] L. BonJour. The structure of empirical knowledge. Harvard University Press, Cambridge, MA, 1985.

[7] L. BonJour. The myth of knowledge. Philosophical Perspectives, 24(1):57-83, 2010.

[8] L. Bovens and S. Hartmann. Bayesian epistemology. Oxford University Press, Oxford, 2003.

[9] L. Bovens and S. Hartmann. Solving the riddle of coherence. Mind, 112(448):601-633, 2003.

[10] L. Bovens and S. Hartmann. Why there cannot be a single probabilistic measure of coherence. Erkenntnis, 63(3):361-374, 2005.

[11] L. Bovens and S. Hartmann. An impossibility result for coherence rankings. Philosophical Studies, 128(1):77-91, 2006.

[12] E. Brendel. Coherence theory of knowledge: A gradational account. Erkenntnis, 50(2-3):293-307, 1999.

[13] R. Carnap. The aim of inductive logic. In E. Nagel, P. Suppes, and A. Tarski, editors, Logic, methodology and philosophy of science, pages 303-318. Stanford University Press, Stanford, CA, 1962.

[14] D. Christensen. Putting logic in its place. Clarendon Press, Oxford, 2004.

[15] B. de Finetti. La prévision: ses lois logiques, ses sources subjectives. Annales de l'Institut Henri Poincaré, 7(1):1-68, 1937.

\footnotetext{
${ }^{21}$ Except perhaps for the requirement that a sufficiently high degree of partial epistemic justification should constitute a approximation - for pragmatic purposes - of full epistemic justification.
} 
[16] B. de Finetti. Teoria delle probabilità: sintesi introduttiva con appendice critica. Volumi primo e secondo. Nuova Biblioteca Scientifica Einaudi. Giulio Einaudi Editore, Turin, 1970.

[17] B. de Finetti. Theory of probability: a critical introductory treatment. Vol. 1. Translated by Antonio Machi and Adrian Smith, with a foreword by D. $V$. Lindley. Wiley Series in Probability and Mathematical Statistics. John Wiley \& Sons, London, New York, Sydney, 1974.

[18] R. Diestel. Graph theory, volume 173 of Graduate Texts in Mathematics. Springer, Heidelberg, 4 edition, 2010.

[19] F. Dietrich and L. Moretti. On coherent sets and the transmission of confirmation. Philosophy of Science, 72(3):403-424, 2005.

[20] G.A. Dirac. Extensions of Menger's theorem. Journal of the London Mathematical Society, 38:148-161, 1963.

[21] G.A. Dirac. Short proof of Menger's graph theorem. Mathematika, 13:42-44, 1966.

[22] I. Douven and W. Meijs. Bootstrap confirmation made quantitative. Synthese, 149(1):97-132, 2006.

[23] K. Easwaran and B. Fitelson. An "evidentialist" worry about Joyce's argument for probabilism. Dialectica, 66(3):425-433, 2012.

[24] S. Fajardo and H.J. Keisler. Model theory of stochastic processes, volume 14 of Lecture Notes in Logic. A.K. Peters, Natick, MA, 2002.

[25] B. Fitelson. A probabilistic theory of coherence. Analysis, 63(3):194-199, 2003.

[26] B. Fitelson and D. McCarthy. Steps toward a new foundation for subjective probability. Work in progress, Munich Center for Mathematical Philosophy, 2012.

[27] R. Foley. Beliefs, degrees of belief, and the Lockean Thesis. In F. Huber and C. Schmidt-Petri, editors, Degrees of Belief, volume 342 of Synthese Library, pages 37-47. Springer, Dordrecht, 2009.

[28] R. Fumerton. Epistemology. Blackwell, Oxford, 2006.

[29] C. Glymour. Theory and Evidence. Princeton University Press, Princeton, NJ, 1980.

[30] T. Grünwald. Ein neuer Beweis eines Mengerschen Satzes. Journal of the London Mathematical Society, 13:188-192, 1938.

[31] A. Hájek. Staying regular? Conference paper, 9th Annual Formal Epistemology Workshop, 2012.

[32] S.O. Hansson and E.J. Olsson. Providing foundations for coherentism. Erkenntnis, 51(2-3):243-265, 1999.

[33] F. Hausdorff. Dimension und äußeres Maß. Mathematische Annalen, 79(12):157-179, 1918.

[34] F.S. Herzberg. A definable nonstandard enlargement. Mathematical Logic Quarterly, 54(2):167-175, 2008.

[35] F.S. Herzberg. Addendum to "A definable nonstandard enlargement". Mathematical Logic Quarterly, 54(6):666-667, 2008.

[36] F.S. Herzberg. The consistency of probabilistic regresses. A reply to Jeanne Peijnenburg and David Atkinson. Studia Logica, 94(3):331-345, 2010.

[37] F.S. Herzberg. The consistency of probabilistic regresses: Some implications for epistemological infinitism. Erkenntnis, 78(2):371-382, 2013.

[38] F.S. Herzberg. The dialectics of infinitism and coherentism: Inferential justification versus holism and coherence. Synthese, 191(4):701-723, 2014.

[39] J.M. Joyce. Accuracy and coherence: Prospects for an alethic epistemology of partial belief. In F. Huber and C. Schmidt-Petri, editors, Degrees of Belief, volume 342 of Synthese Library, pages 263-297. Springer, Dordrecht, 2009. 
[40] V. Kanovei and M. Reeken. Nonstandard analysis, axiomatically. Springer Monographs in Mathematics. Springer, Berlin, 2004.

[41] V. Kanovei and S. Shelah. A definable nonstandard model of the reals. Journal of Symbolic Logic, 69(1):159-164, 2004.

[42] D. König. Über trennende Knotenpunkte in Graphen (nebst Anwendungen auf Determinanten und Matrizen). Acta Litterarum ac Scientiarum (Szeged), 6(2-3):155-179, 1933.

[43] P. Klein and T.A. Warfield. What price coherence? Analysis, 54(3):129-132, 1994.

[44] P. Klein and T.A. Warfield. No help for the coherentist. Analysis, 56(2):118$121,1996$.

[45] K. Lehrer. Theory of knowledge. Westview Press, Boulder, CO, 2000.

[46] H. Leitgeb. Reducing belief simpliciter to degrees of belief. Annals of Pure and Applied Logic, 164(3):1338-1389, 2013.

[47] H. Leitgeb and R. Pettigrew. An objective justification of Bayesianism I. Measuring inaccuracy. Philosophy of Science, 77(2):201-235, 2010.

[48] H. Leitgeb and R. Pettigrew. An objective justification of Bayesianism II. The consequences of minimizing inaccuracy. Philosophy of Science, 77(2):236-272, 2010.

[49] C.I. Lewis. An analysis of knowledge and valuation. Open Court, LaSalle, IL, 1946.

[50] J. Locke. An essay concerning human understanding. Ed. by. P.H. Nidditch. Oxford University Press, Oxford, 1979.

[51] D.C. Makinson. The paradox of the preface. Analysis, 25(6):205-207, 1965.

[52] W. Meijs and I. Douven. On the alleged impossibility of coherence. Synthese, 157(3):347-360, 2007.

[53] K. Menger. Zur allgemeinen Kurventheorie. Fundamenta Mathematica, 10:96$115,1927$.

[54] D.W. Miller. Popper's contributions to the theory of probability and its interpretation. In The Cambridge Companion to Popper. Cambridge University Press, Cambridge, 2014.

[55] L. Moretti and K. Akiba. Probabilistic measures of coherence and the problem of belief individuation. Synthese, 154(1):73-95, 2007.

[56] E.J. Olsson. What is the problem of coherence and truth? Journal of Philosophy, 99(5):246-272, 2002.

[57] E.J. Olsson. Against coherence: Truth, probability, and justification. Oxford University Press, Oxford, 2005.

[58] E.J. Olsson. The impossibility of coherence. Erkenntnis, 63(3):387-412 (2006), 2005.

[59] J.L. Pollock. The paradox of the preface. Philosophy of Science, 53(2):246-258, 1986.

[60] K. Popper and D.W. Miller. Why probabilistic support is not inductive. Philosophical Transactions of the Royal Society of London. Series A. Mathematical and Physical Sciences, 321(1562):569-591, 1987.

[61] W.V.O. Quine and J. Ullian. The web of belief. Random House, New York, 1970.

[62] A. Rényi. On a new axiomatic theory of probability. Acta Mathematica Academiae Scientiarum Hungaricae, 6:285-335, 1955.

[63] A. Robinson. Non-standard analysis. Nederlandse Akademie van Wetenschappen. Proceedings. Series A. Indagationes Mathematicae, 64:432440, 1961. 
[64] A. Robinson. Non-standard analysis. Princeton Landmarks in Mathematics. Princeton University Press, Princeton, NJ, 1996.

[65] J.N. Schupbach. On the alleged impossibility of Bayesian coherentism. Philosophical Studies, 141(3):323-331, 2008.

[66] T. Shogenji. Is coherence truth conducive? Analysis, 59(4):338-345, 1999.

[67] M. Siebel and W. Wolff. Equivalent testimonies as a touchstone of coherence measures. Synthese, 161(2):167-182, 2008.

[68] M.H. Stone. The theory of representations for Boolean algebras. Transactions of the American Mathematical Society, 40(1):37-111, 1936.

[69] P. Walley. Statistical reasoning with imprecise probabilities, volume 42 of Monographs on Statistics and Applied Probability. Chapman and Hall, London, 1991.

[70] P. Walley. Towards a unified theory of imprecise probability. International Journal of Approximate Reasoning, 24(2-3):125-148, 2000.

[71] R. Wedgwood. Outright belief. Dialectica, 66(3):309-329, 2012.

[72] K. Weichselberger. The theory of interval-probability as a unifying concept for uncertainty. International Journal of Approximate Reasoning, 24(2-3):149-170, 2000.

[73] H. Whitney. Congruent graphs and the connectivity of graphs. American Journal of Mathematics, 54(1):150-168, 1932.

[74] L. Wittgenstein. On certainty. Blackwell, Oxford, 1969. 